The Social and Psychological Aspects Behind Flight

Jillian Avis

invoke: Undergraduate Sociology Journal Vol. 1 No. 1 (2012) 
Non-verbal communication commences in infants shortly after birth, and verbal communication often starts before the age of two (Vasta, Younger, Adler, Miller, and Ellis, 2009). Dependency on others is a pivotal characteristic during infancy, however; as infants develop into their adolescent and adult counterparts, independence becomes more prominent. Independence, to a certain degree, is healthy in an adult individual, and while an optimal amount may facilitate communication with peers and coworkers, insufficient or excessive levels may hinder effective communication. The topic of independence and communication pertains directly to aviation - if crew members are overly dependent, they may not challenge the captain when required, and conversely, if other crew members are excessively independent, they may not work collaboratively in a team-oriented setting. By improving interpersonal relationships among crew members, the concept of Crew Resource Management has been increasingly stressed over the years to reduce pilot error (Helmreich, Merritt, and Wilhelm, 1999). Because the importance of effective communication is fundamental to achieving the crew's ultimate goal safely and successfully, the concept of Crew Resource Management (CRM) was established to ensure safe success, especially in the field of aviation.

\section{Crew Resource Management}

In an aviation setting, Crew Resource Management refers to effective teamwork that requires both efficient and effective communication of pertinent information between the flight deck, cabin crew members, and those not on the aircraft but responsible for flight information (e.g. flight dispatchers or air traffic controllers) (Brown and Moren, 2003). More specifically, CRM focuses on a variety of factors that can influence the pilot's and crew members' performance and accuracy. These factors include situational awareness, communication skills, task allocation, and decision making within a collaborative team setting (Jones, 2010). Because numerous aviation disasters have occurred due to team insubordination or a team member's failure to communicate or listen to other members, airlines have increasingly emphasized CRM training over the years. Research has shown that when 
teams practice routine communication and delegation skills during periods of low-stress, there is an increased probability of successful team performance when high-stress emergency situations do occur (Jones, 2010).

\section{The Interview}

In an interview with a retired airline pilot, who flew internationally with five commercial airlines for a total of thirty years, the interviewer administered questions about Crew Resource Management. Themes discussed in the interview such as evolution of CRM pilot attitude towards computer technology and the role of collaboration in emergency situations will be explored throughout this paper. Please see the Appendix for the interview manuscript.

\section{Origins \& Evolution}

What is now regarded as Crew Resource Management originated from a National Aeronautics and Space Administration (NASA) workshop in 1979 entitled Resource Management on the Flightdeck (Helmreich et al., 1999). This workshop was largely a result of recent NASA research which had looked at the causes of aviation accidents. Wiener, Kanki, and Helmreich (1993) identified seven main factors that were common amongst aircraft incidents: preoccupation with minor mechanical problems, inadequate team leadership, failure of the leader (pilot) to delegate tasks, failure of crew members to set priorities, inadequate computer monitoring in the cockpit, failure to utilize available data and failure to communicate near-future plans. Because the meeting revealed that the majority of air crashes were caused by failures of communication, decision making, and leadership, many airlines left the meeting determined to develop training programs that would focus on interpersonal skills between crew members (Helmreich et al., 1999). 
CRM has evolved through four generations since the NASA workshop in 1979. According to Helmreich et al. (Ibid.), Cockpit Resource Management ${ }^{1}$ was first implemented in 1981 (by United Airlines) and focused generally on interpersonal skills and strategies; however, no clear definition of 'appropriate cockpit behaviour' was advised. Success of the Cockpit Resource Management classroom training was limited in the first generation because many traditional pilots felt their personalities were being purposefully manipulated. The second generation of Crew Resource Management focused on a shift from cockpit to crew resource management and specific interpersonal strategies were taught such as team building, situation awareness, and stress management (Ibid.). The third generation (in addition to applying more specific principles) extended education to groups beyond the cockpit, for example, flight attendants, dispatchers, and maintenance workers. Also, pilots were advised to exert a 'leadership' role and not solely a 'pilot' role. Baron (n.d.) mentions that traditionally captains of commercial aircrafts were considered to be God-like figures and the pilot's decision was not to be challenged because their decision was always accurate. It was a marked transition for pilots to descend from the traditional-authoritative role to the newly established team-leader role. Finally, the fourth generation has shown increasing acceptance rates with CRM principles and many of the concepts have become proceduralized by airlines, for example, specific communication strategies may appear on checklists (Helmreich et al., 1999).

Crew Resource Management has evolved immensely since its first implementation in 1981. As Helmreich et al. (Ibid) mention, researchers cannot solely conclude that CRM is effective due to an array of reasons, for example, not all pilots have accepted CRM principles and some pilots who embraced the idea in the classroom may have difficulties applying the principles during flight. Furthermore, because aviation accident rates are already low and training programs vary within the

\footnotetext{
${ }^{1}$ During the first generation, it was denoted by Cockpit Resource Management. The term changed to Crew Resource Management in the second generation to include crew members not in the cockpit, for example flight attendants and air traffic controllers.
} 
airline and between other airlines, it is difficult to calculate strong correlations between CRM training and flight accident rates. Despite that correlational relationships cannot be indefinitely deduced, CRM training has become more sophisticated over the years and has been subjectively stated by many pilots, crew members, and grounded personnel as crucial to their career. Kanki \& Palmer (1993) suggest that researchers should execute more controlled research instead of the traditional self-report questionnaire and independent evaluation method. However, limitations still remain with this approach because it is difficult to fully control for differences in crew coherence and overall flight crew performance (Kanki \& Palmer, 1993).

\section{Aviation}

\section{Communication Errors, Emotion \& Stress}

Evolution from the first to fourth generation of CRM suggests that there are many factors underneath the broad concept of 'communication and interpersonal relations' that have resulted in pilot error. Brown \& Moren (2003) advocate that emotional dynamics can heavily influence crew member communication malfunction. They proposed that airlines should practice new training to specifically address commonly found pilot qualities such as the tendency to avoid self-blame. According to an analysis of the Aviation Safety Reporting System, $70 \%$ of pilot-related errors and accidents were due to erroneous transfers of information between aircraft employees and the most common communication error, at $37 \%$, was failure to initiate the exchange of information (Ibid.). It is not surprising that in order to maintain a successful and safe flight, information needs to exist and it needs to be readily available to those who require it (Billings and Reynard, 1981). If $37 \%$ of pilots, flight attendants or grounded personnel are not even relaying pertinent information to other crew members, then the percentage of crew members who effectively communicate initially has plummeted to $63 \%$. In addition, this $63 \%$ does not entirely consist of crew members who successfully communicate the message onto other crew members. It appears that as a message needs to arrive at an increasing 
number of crew members, errors propagate. Also, if a message needs to be communicated from a cockpit member to flight attendant, there is the physical barrier of the cabin door and misinterpretation and frustration can occur between the pilots and flight attendants (Brown and Moren, 2003). The physical barrier between pilots and flight attendants has only worsened over the years: In 1981 the “sterile cockpit rule" was implemented and after the 9/11 attacks in 2011 cockpit doors were physically strengthened (Chute and Wiener, 1995). In addition to the physical barrier of the cabin door, Chute \& Wiener (Ibid.) note that previous literature has divided the cockpit (pilot and first officer) and flight crew (flight attendants) into 'two cultures' because of male- and female-dominance, respectively.

Various emotions are evident in interpersonal relationships between crew members, especially within the cockpit. Emotions such as excitement or shame are common and are often sources of stress for crew members. In the cockpit, there are at least four causes of shame as explained by Brown and Moren (2003). They include:

i. relating to an unfamiliar crew member

a. crew scheduling changes on a monthly basis for most airlines; this indicates that up to twelve times per year, crew members may experience shame and subsequent stress

ii. subordinates interacting with overly-confident or "cocky" personality types, qualities more likely to be found in the pilot

a. subordinates and trainees may feel excess pressure to remain 'error-free' when working with an intimidating pilot and, in situations of error, this may induce shame and stress

iii. miscellaneous mistakes in behaviour

In general, feelings of shame affect can be induced by unfamiliar employees, new situations, and differences perceived in authority, status, gender, and age amongst other factors ( Ibid.). It should be noted that shame is only one emotion that crew members may experience; other feelings such as 
excitement, inhibition, and awkwardness have the potential to adversely affect effective communication and accurate job performance.

\section{Attitude}

In the administered interview with Captain G. Avis, it was evident that he believed pilot attitudes differed toward the increasing incorporation of computer technology in the cockpit. Before integration of computer technology, pilots were physically engaged in flying the aircraft typically with a 'stick-shift'. It is therefore understandable that pilots may have been initially skeptical about the computer system and its ability to perform a complicated human duty. In a 1982 study, a questionnaire was designed to measure attitudes about issues in crew management. To date, more than six hundred airline pilots have completed this questionnaire with results revealing that attitudes differ significantly as a function of status (e.g. captain, first officer) (Helmreich, Foushee, Benson, and Russini, 1986).

Helmreich et al. (Ibid.) utilized the 1982 survey to assess attitudes from 114 pilots and 5 'check airmen'2 that evaluated flight crew performance. In other words, this experiment attempted to establish a link between pilot self-reports and independent evaluations by 'check airmen'. Pilots who rated average on the Likert scale by check airmen were not included in the study; therefore, pilots in the study were on either ends of the spectrum: 'extremely poor' or 'outstanding' in terms of performance. Results revealed that a correlation between self-reports and independent evaluations do exist. In selfreports, pilots who displayed recognition of capability limitations and demonstrated encouragement of other crew members to question the pilot's decision, tended to be independently evaluated by check air men as 'above average' or 'excellent'. Conversely, self-reports of pilots who demonstrated that they did not recognize personal limitations, were less sensitive to other crew member's reactions, and employed authoritarian management styles, were rated by air checkmen as either 'below average' or 'poor' (Ibid.). It is evident that crew members may perceive and believe the pilot to be excellent if

\footnotetext{
${ }^{2}$ Air checkmen only rated pilots whom they had directly worked with. For each pilot, two check airmen completed evaluations to establish inter-rater reliability.
} 
effective communication and delegation skills have been previously employed. Therefore, crew members attitudes towards specific pilots, whom they believe to be excellent, may induce a more positive work environment. Ultimately, attitudes toward the pilot can render a team that effectively uses Crew Resource Management principles. Future suggestions to increase effective use of CRM could include biweekly meetings for crew members to discuss communication strengths and weaknesses of the team.

\section{Cultural Differences}

Because different cultures value certain characteristics over others, for example, submissiveness or assertiveness, it is expected that communication between crew members may differ between airlines companies. Cross-culturally, research has demonstrated that nearly $70 \%$ of aircraft accidents are due to non-technical skills regarding ineffective communication, lack of situational awareness, weak team work, and poor decision making (Sekurli and Gerede, 2011). Although technical training focusing on human-technology interaction consumes the majority of time for airline employees, CRM training is pertinent because it focuses solely on human-human interaction. Internationally, airlines have implemented training courses for employees on CRM; however, there continues to be a general dissatisfaction from non-American airlines - classroom training does not necessarily translate to effective communication in-flight.

Sekurli and Gerede (Ibid.) suggest that standard CRM classroom training does not address cultural differences that may exist both between and within countries. American culture tends to value independence, autonomy, and a free-market economy; therefore, CRM principles tailored to American airlines may not be effective in other countries such as Turkey or the Philippines. In a recent study Sekurli \& Gerede (Ibid.) performed a questionnaire to address cultural differences between $350 \mathrm{crew}$ members including pilots, first officers, and second officers. Four scales in relation to culture differences were assessed: "communication", "giving orders", "perception of stress", and "obeying the 
rules" (Ibid.). Turkey's history of domestic market deregulation (which caused a spontaneous increase in airline demand) and pilots with differing educational backgrounds were issues the researchers took into account. The questionnaires revealed that employees with professional education valued clear communication, employees from patriarchical cultures were reluctant to express opinions, and employees with military education noted importance of a hierarchy within the crew. It can be concluded from this study that universal CRM training programs are not effective in addressing cultural differences (Ibid.). To increase the use of CRM principles in aviation cross-culturally, CRM training programs should be tailored accordingly to different groups of people.

Seva, Gutierrez, Duh and Chong (2007) analyze the term 'culture' and its influence on behaviour in the cockpit. Culture can be broken down into three categories: national, professional and organizational. National refers to beliefs and behaviours that are distinct to a country, professional refers to background education and current practice of a specific profession, and organizational refers to behaviours or beliefs due to membership in a group such as a specific airline company (Ibid.). By using a questionnaire, 88 male captains and first officers from four Filipino airline companies were asked 96 items to assess CRM concepts such as communication, situational awareness, teamwork, workload management, decision making, and culture. Similar results to previous research revealed that, on a national level, individuals from 'passive-dependent' cultures (such as Filipinos, Taiwanese, and Indians) tended to accept authority most willingly and were afraid to question superior crew members (Ibid.). Results were most significant on an organizational level: employees of well-established airline companies appeared more strongly committed to CRM principles - this is likely because the airline had previously communicated strong support toward CRM. The outcomes of this study revealed that airline attitude toward a specific principle, for example CRM, is influential on employees and their subsequent beliefs and behaviours. 
Helmreich et al. (1999) also suggest that culture can heavily impact the effectiveness of CRM principles. Latin American countries and China are referred to as 'High Power Distance' cultures in which authoritarianism is valued and subordinates may be reluctant to question pilot decisions or actions because they do not want to show disrespect (Ibid.). However, these cultures do accept the importance of teamwork and working effectively together. On the other hand, the United States of America is referred to as a 'Highly Individualistic' culture: the 'drongo' pilot and subordinate team may not work together as an effective team because specific members are excessively independent. Other countries, such as Greece and Korea, are referred to as 'High Uncertainty Avoidance' cultures in which CRM principles are gladly welcomed because teamwork is deemed very important (Ibid.).

\section{Evaluation \& Effectiveness}

Crew Resource Management has evolved from introducing training programs, to CRM training program evaluation, and most currently, to evaluating pilot human interaction skills (Flin \& Martin, 2001). Evaluation of CRM training program commenced when the Advanced Qualification Program (AQP) was adopted by numerous U.S. airline companies - airlines were obligated to evaluate both technical and non-technical training in simulation training (Ibid.). In the interview with captain G. Avis, he mentioned that toward the end of his career simulator training and testing increasingly adapted non-technical skills. He explained that this simulator training was administered to ensure the pilot and first-officer were performing an optimal number of tasks, but were not individually overloaded.

Establishing program effectiveness of CRM is a difficult task. As mentioned before, there are multiple factors that are involved in CRM effectiveness such as applicability from classroom to inflight, pilot attitudes, and numerous other variables that may affect flight crew performance. A recent meta-analysis of sixteen CRM evaluation studies has revealed hopeful results of CRM training. The largest positive effects of CRM training were demonstrated in crew attitudes and behaviours, while a medium effect was found for knowledge (O’Connor, Campbell, Newon, Melton, Salas, and Wilson, 
2008). It was also revealed that participants had an overall positive attitude toward CRM training with a mean of 4 out of 5 on a Likert scale. If CRM methods are to be increasingly incorporated into other career fields, such as medicine, future research needs to address variables that may increase knowledge and apply tactics to reduce individual status differences to a minimal level.

Although there is an abundance of correlational research regarding CRM training and its effectiveness on reducing pilot error, there is a paucity of experimental research on the effectiveness of CRM. According to Sauer, Darioly, Mast, Schmid and Bischof (2010), the main objective of CRM training is to provide crew members with good communication skills including receptiveness and influencing skills. In the current experimental two-by-two study, 64 males with similar post-secondary education were randomly assigned to a group that either did or did not provide communication skills training and a group that was either hierarchical in nature or of congruence. All subjects were placed in a Cabin Air Management System, which requires subjects to control a mimicked spacecraft life support system (Ibid.). Amongst numerous findings, the most significant finding was that communication skills training benefitted the hierarchical group most. The researchers suggested future research should perform a multi-level analyses to evaluate the effectiveness of CRM training in specific career fields.

\section{Future Implications}

In-class training can only be effective up to certain extent - inevitably, there will be employees that cannot attend scheduled dates. Online education offers several alternatives to in-class training: considerable cost savings and a higher number of employees are reached because online training can be offered across a large geographic span and it can be completed at a time convenient for the employee (Kearns, 2011). However, it should be mentioned that in-class training often engages in social activities such as game-playing role exercises. If Crew Resource Management training were to be implemented as an online tutorial, research would have to establish social interaction equivalents to the online version. Kearns (Ibid.) developed a one-variable, multiple condition study in which 36 pilots 
were placed into one of three groups: a single-pilot resource management (SRM) ${ }^{3}$ group with hands-on practice, an SRM group with guided mental practice, and a control group that received no training. Mental workload performance and situational awareness were the dependent variables - a secondary task assessment and global task assessments were used to measure the variables, respectively (Ibid.). Results revealed that there were no significant differences between either practice conditions (hands-on practice or mental practice) because each group exhibited improved situation awareness. There was, however, a significant difference between these two practice conditions and the group that did not receive any training (Ibid.). This research suggests that "guided mental practice", which would be equivalent to an online tutorial, is equally effective in teaching CRM principles as "hands-on practice". In the future, if both in-class and online tutorials for CRM are available to airline employees, there would be a host of benefits.

As noted by Lavitt (1992), CRM in-class training is continually evolving to include new material. For example, the Flight Safety Philadelphia Learning Centre updated their curriculum in 1992 to include tropical weather. This update was intended to educate flight crew about past tropical weather aviation accidents and teach the crew how to react to an emergency as a team. The increasing number of added courses to CRM curriculum will never cease and if airlines offered pilots and flight crew the option to engage in online courses, more employees over a large geographic span could be educated.

\section{Application to Other Fields}

\section{Medicine}

Comparable to an aircraft, a hospital is an interacting dynamic of relationships between humans and machines, and between humans and humans (Doucette, 2006). Like flight crew, health care professionals such as nurses or doctors must establish effective working relationships in order to

\footnotetext{
${ }^{3}$ Single Pilot Resource Management is an adaptation of Crew Resource Management, in which CRM concepts are taught to single pilot operators (Kearns, 2011)
} 
deliver safe and successful patient care. For example, situation awareness is a fundamental concept addressed in aviation CRM. By educating healthcare professionals about situation awareness, hospitals could decrease the chance that factors, such as poor communication or inability to challenge superiors, will not overload or distract employees and ultimately result in medical error. Largely to this day, the main difference between aviation and medicine include errors due to fatigue, stress, and inadequate communication that are chiefly accounted for by the airline industry. Because of this, these concepts are used for training and improvements. Conversely, in the medical field, pressure exists to conceal mistakes, and many health care professionals even report that error is handed inappropriately in their own hospital (Sexton, Thomas and Helmriech, 2000).

In a recent publication, researchers adapted CRM aviation techniques to a pediatric critical care unit at the Children's Hospital in Philadelphia. With the assistance of pilots, who had extensive experience in the field of aviation CRM, training was delivered to a total 120 health care professionals (Ryan, Mericle, Frush, Alton, and Meliones, 2008). The goals of training were to educate employees in the critical care unit about communication, situation awareness, and effective use of team language. Self-report surveys completed after a series of 3-hour training periods revealed an improvement of how staff perceived collaboration in the team. Similarly, independent evaluations of staff demonstrated an increased engagement in effective teamwork (Ibid.).

\section{Dentistry}

Dentistry is another healthcare field that benefits from the adaptation of CRM principles. Threat and error management techniques (TEM) are "forward-thinking" strategies taught to dentists, nurses, and dental hygienists (Pinsky, Taichman, and Sarment, 2010). These techniques are used to assess risk for medical error in high-stress situations. Employees are better able to assess potential for dental error because situation awareness increases when "risk analysis" is employed - an adaptation of CRM used in aviation. 